\title{
On the Error Bound in the Normal Approximation for Jack Measures
}

\author{
LOUIS H. Y. CHEN ${ }^{1}$ MARTIN RAIČ ${ }^{2}$ and LÊ VĂN THÀNH ${ }^{3}$ \\ ${ }^{1}$ Department of Mathematics, National University of Singapore, 10 Lower Kent Ridge \\ Road, Singapore 119076. e-mail: matchyl@nus.edu.sg \\ ${ }^{2}$ University of Ljubljana, University of Primorska, and Institute of Mathematics, Physics \\ and Mechanics, Slovenia. e-mail: martin.raic@fmf.uni-lj.si \\ ${ }^{3}$ Department of Mathematics, Vinh University, 182 Le Duan, Vinh, Nghe An, Vietnam. \\ e-mail: levt@vinhuni.edu.vn
}

\begin{abstract}
In this paper, we obtain uniform and non-uniform bounds on the Kolmogorov distance in the normal approximation for Jack deformations of the character ratio, by using Stein's method and zero-bias couplings. Our uniform bound comes very close to that conjectured by Fulman [J. Combin. Theory Ser. A, 108 (2004), 275-296]. As a by-product of the proof of the non-uniform bound, we obtain a Rosenthal-type inequality for zero-bias couplings.
\end{abstract}

Keywords and phrases: Stein's method, zero-bias coupling, Jack measure, Jack deformation, Kolmogorov distance, uniform bound, non-uniform bound, rate of convergence.

MSC 2010 subject classifications: Primary 60F05; Secondary 60C05

\section{Introduction and main results}

Let $G$ be a finite group, and $G^{*}$ the set of all the irreducible representations of $G$. Then

$$
\sum_{\pi \in G^{*}} \operatorname{dim}(\pi)^{2}=|G|
$$

where $\operatorname{dim}(\pi)$ denotes the dimension of the irreducible representation $\pi$ (see (Sagan, 2001, Proposition 1.10.1)). The Plancherel measure is a probability measure on $G^{*}$ defined by

$$
\mathbb{P}(\{\pi\})=\frac{\operatorname{dim}(\pi)^{2}}{|G|}
$$

Let $n$ be a positive integer. An important special case is the finite symmetric group $\mathcal{S}_{n}$. For this group, the irreducible representations are parametrized by partitions $\lambda$ of $n$, and the dimension of the representation associated to $\lambda$ is known to be equal to the number of standard $\lambda$-tableaux (see (Sagan, 2001, Theorem 2.6.5)). We also denote the number of standard $\lambda$-tableaux by $\operatorname{dim}(\lambda)$, and write a partition $\lambda=\left(\lambda_{1}, \lambda_{2}, \ldots, \lambda_{m}\right)$ of $n$ simply $\lambda \vdash n$. The hooklength of a box $s$ in the partition $\lambda$ is defined as $h(s)=a(s)+l(s)+1$. Here $a(s)$ denotes 
the number of boxes in the same row of $s$ and to the right of $s$ (the "arm" of $s$ ) and $l(s)$ denotes the number of boxes in the same column of $s$ and below $s$ (the "leg" of $s)$. The Plancherel measure in this case is

$$
\mathbb{P}(\{\lambda\})=\frac{\operatorname{dim}(\lambda)^{2}}{n !}
$$

By the hook formula (see, e.g., Sagan (2001)) which states that

$$
\operatorname{dim}(\lambda)=\frac{n !}{\Pi_{s \in \lambda} h(s)},
$$

where the product is over boxes in the partition and $h(s)$ is the hooklength of a box $s$, we also have

$$
\mathbb{P}(\{\lambda\})=\frac{n !}{\Pi_{s \in \lambda} h^{2}(s)} .
$$

A random partition $\lambda$ chosen by the Plancherel measure has interesting connections to the Gaussian unitary ensemble (GUE) of random matrix theory. We recall that the joint probability density of the eigenvalues $x_{1} \geq x_{2} \geq \cdots \geq x_{n}$ of the Gaussian orthogonal ensemble (GOE), Gaussian unitary ensemble (GUE), and Gaussian symplectic ensemble (GSE) is given by

$$
\frac{1}{Z_{\beta}} \exp \left(-\frac{x_{1}^{2}+\cdots+x_{n}^{2}}{2}\right) \Pi_{1 \leq i<j \leq n}\left(x_{i}-x_{j}\right)^{\beta}
$$

with $\beta=1,2,4$, respectively. Here $Z_{\beta}$ is a normalization constant. Let $\pi$ be a permutation chosen from the uniform measure of the symmetric group $\mathcal{S}_{n}$ and $l(\pi)$ the length of the longest increasing subsequence in $\pi$. Baik, Deift, and Johansson (1999) proved that $(l(\pi)-2 \sqrt{n}) / n^{1 / 6}$ converges to the Tracy-Widom distribution as $n \rightarrow \infty$. It follows from the Robinson-Schensted-Knuth correspondence (see Sagan (2001)) that the first row of a random partition distributed according to the Plancherel measure has the same distribution as the longest increasing subsequence of a random permutation distributed according to the uniform measure. So the result of Baik, Deift, and Johansson (1999) says that a suitably normalized length of the first row of a random partition distributed according to the Plancherel measure converges to the Tracy-Widom distribution. Borodin, Okounkov, and Olshanski (2000), Johansson (2001) proved that the joint distribution of suitably normalized lengths of the rows of a random partition distributed according to the Plancherel measure converges to the joint distribution of the eigenvalues $x_{1} \geq x_{2} \geq \cdots \geq x_{n}$ of a $n \times n$ GUE matrix.

$\mathrm{Jack}_{\alpha}$ measure is an extension of the Plancherel measure. For $\alpha>0$, the $\mathrm{Jack}_{\alpha}$ measure is a probability measure on the set of all partitions of a positive integer $n$, which chooses a partition $\lambda$ with probability

$$
\mathbb{P}_{\alpha}(\{\lambda\})=\frac{\alpha^{n} n !}{\Pi_{s \in \lambda}(\alpha a(s)+l(s)+1)(\alpha a(s)+l(s)+\alpha)},
$$


where the product is over all boxes in the partition. For example, the partition

$$
\lambda=\square
$$

of 6 has $\mathrm{Jack}_{\alpha}$ measure

$$
\frac{720 \alpha^{3}}{(3 \alpha+2)(2 \alpha+3)(\alpha+2)^{2}(2 \alpha+1)^{2}} \text {. }
$$

We notice that the Jack measure with parameter $\alpha=1$ agrees the Plancherel measure of the symmetric group since it coincides with (1.1). It is mentioned in Matsumoto (2008) that for any positive real number $\beta>0$, the $\mathrm{Jack}_{\alpha}$ measure with $\alpha=2 / \beta$ is the counterpart of the Gaussian $\beta$-ensemble $(G \beta E)$ with the probability density function proportional to (1.2).

Let $\lambda$ be a partition of $n$ chosen from the Plancherel measure of the symmetric group $\mathcal{S}_{n}$, and $\chi^{\lambda}(12)$ the character of the irreducible representation associated to $\lambda$ evaluated on the transposition (12). Characters of the irreducible representations of a symmetric group are of interest in the literature because they play central roles in representation theory and other fields of mathematics such as random walks (Diaconis and Shahshahani (1981)) and the moduli space of curves (Eskin and Okounkov (2001)). The quantity $\chi^{\lambda}(12) / \operatorname{dim}(\lambda)$, which is a normalization of $\chi^{\lambda}(12)$, is called a character ratio. As $\lambda$ is distributed according to the Plancherel measure, $\chi^{\lambda}(12)$ is a random variable.

In Kerov (1993), it is stated that

$$
\frac{\sqrt{\left(\begin{array}{l}
n \\
2
\end{array}\right)} \chi^{\lambda}(12)}{\operatorname{dim}(\lambda)}
$$

is asymptotically normal with mean 0 and variance 1 as $n \rightarrow \infty$. A proof of Kerov's central limit theorem can be found in Hora (1998), which uses the method of moments and combinatorics. More recently, a proof in Śniady (2006) uses the genus expansion of random matrix theory, and another in Hora and Obata (2007) uses quantum probability.

By a formula due to Frobenius (1900) (see also Fulman (2006)), we have

$$
\frac{\chi^{\lambda}(12)}{\operatorname{dim}(\lambda)}=\frac{1}{\left(\begin{array}{l}
n \\
2
\end{array}\right)} \sum_{i}\left(\left(\begin{array}{c}
\lambda_{i} \\
2
\end{array}\right)-\left(\begin{array}{c}
\lambda_{i}^{\prime} \\
2
\end{array}\right)\right) .
$$

Now, for $\alpha>0$, the random variable we will study in this paper is

$$
W_{n, \alpha}=W_{n, \alpha}(\lambda)=\frac{\sum_{i}\left(\alpha\left(\begin{array}{c}
\lambda_{i} \\
2
\end{array}\right)-\left(\begin{array}{c}
\lambda_{i}^{\prime} \\
2
\end{array}\right)\right)}{\sqrt{\alpha\left(\begin{array}{c}
n \\
2
\end{array}\right)}},
$$

where $\lambda$ is chosen from the $\mathrm{Jack}_{\alpha}$ measure on partitions of a positive integer $n$, $\lambda_{i}$ is the length of the $i$-th row of $\lambda$ and $\lambda_{i}^{\prime}$ is the length of the $i$-th column of 
$\lambda$. By (1.4), $W_{n, \alpha}$ coincides with (1.3) when $\alpha=1$. Therefore, the value $W_{n, \alpha}$ is regarded as a Jack deformation of the character ratio. Moreover, as remarked by Fulman (2004), when $\alpha=2, W_{n, 2}$ is the value of a spherical function corresponding to the Gelfand pair $\left(S_{2 n}, H_{2 n}\right)$, where $H_{2 n}$ is the hyperoctahedral group of size $2^{n} n$ !.

Normally approximation for $W_{n, \alpha}$ has been studied by Fulman $(2004,2006)$, Shao and Su (2006), and Fulman and Goldstein (2011) by using Stein's method (see, e.g., Stein (1986)). In Fulman (2004), the author proved that for any fixed $\alpha \geq 1$,

$$
\sup _{x \in \mathbb{R}}\left|\mathbb{P}_{\alpha}\left(W_{n, \alpha} \leq x\right)-\Phi(x)\right| \leq \frac{C_{\alpha}}{n^{1 / 4}},
$$

where $C_{\alpha}$ is a constant depending only on $\alpha, \Phi(x)=\frac{1}{\sqrt{2 \pi}} \int_{-\infty}^{x} \exp \left(-t^{2} / 2\right) \mathrm{d} t$ is the distribution function of the standard normal distribution.

The bound $C_{\alpha} n^{-1 / 4}$ was later improved in Fulman (2006) to $C_{\alpha} n^{-1 / 2}$ using an inductive approach to Stein's method. We note that in all these results, $\alpha>0$ is fixed, but we do not know how $C_{\alpha}$ depends on $\alpha$. An explicit constant is obtained by Shao and $\mathrm{Su}$ (2006) only when $\alpha=1$. More precisely, when $\alpha=1$, Shao and Su (2006) obtained the rate $761 n^{-1 / 2}$ by using Stein's method for exchangeable pairs. More recently, Dołęga and Féray (2016) proved the Berry-Esseen bound for the multivariate case with rate $C_{\alpha} n^{-1 / 4}$, and Dołęga and Śniady (2019) proved a general multivariate central limit theorem for the case where $\alpha=\alpha(n)$ varying with $n$, satisfying

$$
\frac{-\sqrt{\alpha}+1 / \sqrt{\alpha}}{\sqrt{n}}=g_{1}+\frac{g_{2}}{\sqrt{n}}+o\left(\frac{1}{\sqrt{n}}\right),
$$

where $g_{1}$ and $g_{2}$ are constants.

Fulman (2004) conjectured that for general $\alpha \geq 1$, the correct bound is a universal constant multiplied by $\max \left\{\frac{1}{\sqrt{n}}, \frac{\sqrt{\alpha}}{n}\right\}$. While this bound was conjectured for the Kolmogorov distance in (1.6), using Stein's method and zerobias couplings, Fulman and Goldstein (2011) proved that it is indeed the correct bound for the Wasserstein distance for $W_{n, \alpha}$. By the result in Fulman and Goldstein (2011), the central limit theorem for $W_{n, \alpha}$ holds for $\alpha=\alpha(n)$ varying with $n$ as long as $\sqrt{\alpha} / n \rightarrow 0$. As observed by Fulman (2004), this is necessary for $W_{n, \alpha}$ to be asymptotically normal. The bound conjectured by Fulman (2004) for the Kolmogorov distance remains unsolved as bounds on the Kolmogorov distance are usually harder to obtain than bounds on the Wasserstein distance. This paper is an attempt to prove the conjecture of Fulman (2004) for the Kolmogorov distance. We use Stein's method and zero-bias couplings to obtain both uniform and non-uniform error bounds on the Kolmogorov distance for $W_{n, \alpha}$. We have obtained a uniform error bound which comes very close to that conjectured by Fulman (2004). Besides, we have obtained a very small constant. As a by-product of the proof of the non-uniform bound, we obtain a Rosenthal-type inequality for zero-bias couplings. 
Throughout this paper, $Z$ denotes the standard normal random variable and $\Phi(x)=\frac{1}{\sqrt{2 \pi}} \int_{-\infty}^{x} \exp \left(-t^{2} / 2\right) \mathrm{d} t$ its distribution function. For a positive number $x, \log x$ denotes the natural logarithm of $x$, and $\lfloor x\rfloor$ denotes the greatest integer number that is less than or equal to $x$. For a set $S$, the indicator function of $S$ is denoted by $\mathbf{1}(S)$ and the cardinality of $S$ denoted by $|S|$. For $p \geq 1$ and a random variable $X,\left(\mathbb{E}|X|^{p}\right)^{1 / p}$ is denoted by $\|X\|_{p}$. The symbol $C_{p}$ denotes a generic positive constant which is bounded by $B^{p}$ for some universal constant $B$, but can be different for each appearance. We denote $\mathrm{Jack}_{\alpha}$ measure by $\mathbb{P}_{\alpha}$.

Theorem 1.1. Let $n \geq 3$ be an integer. Let $\alpha>0$ and $W_{n, \alpha}$ be as in (1.5). Then

$$
\sup _{x \in \mathbb{R}}\left|\mathbb{P}_{\alpha}\left(W_{n, \alpha} \leq x\right)-\Phi(x)\right| \leq 8.2 \max \left\{\frac{1}{\sqrt{n}}, \frac{\max \{\sqrt{\alpha}, 1 / \sqrt{\alpha}\} \log n}{n}\right\} .
$$

Remark 1.2. If $\frac{\log ^{2} n}{n} \leq \alpha \leq \frac{n}{\log ^{2} n}$, then the bound in Theorem 1.1 is $\frac{8.2}{\sqrt{n}}$. For $\alpha \geq 1$, the bound in Theorem 1.1 is $8.2 \max \left\{\frac{1}{\sqrt{n}}, \frac{\sqrt{\alpha} \log n}{n}\right\}$, which is very close to that conjectured by Fulman (2004).

We prove Theorem 1.1 by using Stein's method for zero bias couplings. Nonuniform bounds on the Kolmogorov distance in the normal approximation for independent random variables using Stein's method were first investigated by Chen and Shao (2001). Stein's method has also been used to study non-uniform bounds on the Kolmogorov distance (Chen and Shao (2004)) and concentration inequalities (Chatterjee and Dey (2010)) for dependent random variables. The method developed in this paper also allows us to obtain a non-uniform bound on the Kolmogorov distance, which we state in the following theorem.

Theorem 1.3. Let $n \geq 3$ be an integer. Let $p \geq 2,1 / n^{2}<\alpha<n^{2}$ and $W_{n, \alpha}$ be as in (1.5). Then for all $x \in \mathbb{R}$, we have

$\left|\mathbb{P}_{\alpha}\left(W_{n, \alpha} \leq x\right)-\Phi(x)\right| \leq \frac{C_{p}}{1+|x|^{p}}\left(\frac{p^{2}}{\log p}\right)^{p} \max \left\{\frac{1}{\sqrt{n}}, \frac{\max \{\sqrt{\alpha}, 1 / \sqrt{\alpha}\} \log n}{n}\right\}$.

Remark 1.4. If $\alpha \geq n^{2}$ and $p \geq 2$, then it will be shown in the appendix that

$$
\mathbb{E}\left|W_{n, \alpha}\right|^{p} \leq C_{p}\left(\frac{p^{2}}{\log p}\right)^{p}\left(\frac{\sqrt{\alpha}}{n}\right)^{p-2} .
$$

Therefore, by applying Markov's inequality, $\left|\mathbb{P}_{\alpha}\left(W_{n, \alpha} \leq x\right)-\Phi(x)\right|$ is bounded by

$$
\frac{C_{p}}{1+|x|^{p}}\left(\frac{p^{2}}{\log p}\right)^{p}\left(\frac{\sqrt{\alpha}}{n}\right)^{p-2}
$$




\section{A Rosenthal-type inequality for zero-bias couplings}

It was shown in Goldstein and Reinert (1997) that for any mean zero random variable $W$ with positive finite variance $\sigma^{2}$, there exists a random variable $W^{*}$ which satisfies

$$
\mathbb{E} W f(W)=\sigma^{2} \mathbb{E} f^{\prime}\left(W^{*}\right)
$$

for all absolutely continuous $f$ with $\mathbb{E}|W f(W)|<\infty$. The random variable $W^{*}$ and its distribution are called $W$-zero biased. Goldstein and Reinert (1997) (see also in (Chen, Goldstein and Shao, 2011, Proposition 2.1)) showed that the distribution of $W^{*}$ is absolutely continuous with the density $g(x)=\mathbb{E}[W \mathbf{1}(W>$ $x)] / \sigma^{2}$.

In this section, we prove a Rosenthal-type inequality for zero-bias couplings, which we state as a proposition below. We will show later that this proposition can be applied to obtain the Rosenthal inequality for sums of independent random variables. The use of a Rosenthal-type inequality is crucial for obtaining a non-uniform bound on the Kolmogorov distance.

Proposition 2.1. Let $W$ be a random variable with mean zero and variance $\sigma^{2}>0$ and let $W^{*}$ be $W$-zero biased. Assume that $W$ and $W^{*}$ are defined on the same probability space. Let $T=W^{*}-W$. Then for every $p \geq 2$,

$$
\mathbb{E}|W|^{p} \leq \kappa_{p}\left(\sigma^{p}+\sigma^{2} \mathbb{E}|T|^{p-2}\right),
$$

where

$$
\kappa_{p}=\frac{(\log 8)^{3}}{196}\left(\frac{7 p}{4 \log p}\right)^{p} .
$$

Proof. Let

$$
f(x)= \begin{cases}x^{p-1} & \text { if } x \geq 0 \\ -(-x)^{p-1} & \text { if } x<0 .\end{cases}
$$

Then $f^{\prime}(x)=(p-1)|x|^{p-2}$ and $x f(x)=|x|^{p}$.

If $2 \leq p \leq 4$, then

$$
\begin{aligned}
\mathbb{E}|W|^{p} & =\mathbb{E} W f(W)=\sigma^{2} \mathbb{E} f^{\prime}(W+T) \\
& =\sigma^{2}(p-1) \mathbb{E}|W+T|^{p-2} \\
& \leq \sigma^{2}(p-1) \max \left\{1,2^{p-3}\right\}\left(\mathbb{E}|W|^{p-2}+\mathbb{E}|T|^{p-2}\right) \\
& \leq \sigma^{2}(p-1) \max \left\{1,2^{p-3}\right\}\left(\sigma^{p-2}+\mathbb{E}|T|^{p-2}\right) \\
& =(p-1) \max \left\{1,2^{p-3}\right\}\left(\sigma^{p}+\sigma^{2} \mathbb{E}|T|^{p-2}\right) .
\end{aligned}
$$

Elementary calculus shows that

$$
(p-1) \max \left\{1,2^{p-3}\right\} \leq \frac{(\log 8)^{3}}{196}\left(\frac{7 p}{4 \log p}\right)^{p}
$$

for all $2 \leq p \leq 4$. Therefore, from (2.4), we see that (2.2) holds for all $2 \leq p \leq 4$.

imsart-generic ver. 2014/10/16 file: 2020.06.14.Jack_measures.tex date: June 16, 2020 
If $p>4$, by Jensen's inequality, we have for all $0<\theta<1$,

$$
\begin{aligned}
\mathbb{E}|W|^{p} & =\mathbb{E} W f(W)=\sigma^{2} \mathbb{E} f^{\prime}(W+T) \\
& =\sigma^{2}(p-1) \mathbb{E}|W+T|^{p-2} \\
& \leq \sigma^{2}(p-1)\left(\theta \mathbb{E}\left(\frac{|W|}{\theta}\right)^{p-2}+(1-\theta) \mathbb{E}\left(\frac{|T|}{1-\theta}\right)^{p-2}\right) \\
& =\sigma^{2}(p-1)\left(\frac{\mathbb{E}|W|^{p-2}}{\theta^{p-3}}+\frac{\mathbb{E}|T|^{p-2}}{(1-\theta)^{p-3}}\right) .
\end{aligned}
$$

By using following inequality

$$
x^{\alpha} y^{1-\alpha} \leq x+y \text { for all } 0<\alpha<1, x \geq 0, y \geq 0,
$$

we have

$$
\begin{aligned}
\mathbb{E}\left(\sigma^{2}|T|^{p-4}\right) & =\mathbb{E}\left(\left(\sigma^{p-2}\right)^{\frac{2}{p-2}}\left(|T|^{p-2}\right)^{\frac{p-4}{p-2}}\right) \\
& \leq \mathbb{E}\left(\sigma^{p-2}+|T|^{p-2}\right) \\
& =\sigma^{p-2}+\mathbb{E}|T|^{p-2} .
\end{aligned}
$$

For the case where $4<p \leq 6,(2.4)$ and (2.7) yield

$$
\begin{aligned}
\mathbb{E}|W|^{p-2} & \leq(p-3) \max \left\{1,2^{p-5}\right\}\left(\sigma^{p-2}+\sigma^{2} \mathbb{E}|T|^{p-4}\right) \\
& \leq(p-3) \max \left\{1,2^{p-5}\right\}\left(2 \sigma^{p-2}+\mathbb{E}|T|^{p-2}\right) .
\end{aligned}
$$

By letting $\theta:=\theta_{1}=1 / 2$, we have from (2.5) that

$$
\mathbb{E}|W|^{p} \leq \sigma^{2}(p-1) 2^{p-3}\left(\mathbb{E}|W|^{p-2}+\mathbb{E}|T|^{p-2}\right) .
$$

Combining (2.9) and (2.8), we obtain

$$
\mathbb{E}|W|^{p} \leq(p-1)(p-3) 2^{p-2} \max \left\{1,2^{p-5}\right\}\left(\sigma^{p}+\sigma^{2} \mathbb{E}|T|^{p-2}\right) .
$$

Numerical calculations show that

$$
(p-1)(p-3) 2^{p-2} \max \left\{1,2^{p-5}\right\} \leq \frac{(\log 8)^{3}}{196}\left(\frac{7 p}{4 \log p}\right)^{p}
$$

for all $4<p \leq 6$. Therefore, from (2.10), we see that (2.2) holds in this case.

For the case where $6<p \leq 8,(2.7)$ and (2.10) yield

$$
\mathbb{E}|W|^{p-2} \leq(p-3)(p-5) 2^{p-4} \max \left\{1,2^{p-7}\right\}\left(2 \sigma^{p-2}+\mathbb{E}|T|^{p-2}\right) .
$$

By letting $\theta:=\theta_{2}=2 / 3$, we have from (2.5) that

$$
\mathbb{E}|W|^{p} \leq \sigma^{2}(p-1)\left(\frac{3}{2}\right)^{p-3}\left(\mathbb{E}|W|^{p-2}+2^{p-3} \mathbb{E}|T|^{p-2}\right) .
$$


Combining (2.11) and (2.12), we obtain

$$
\mathbb{E}|W|^{p} \leq(p-1)(p-3)(p-5) 3^{p-3} \max \left\{1,2^{p-7}\right\}\left(\sigma^{p}+\sigma^{2} \mathbb{E}|T|^{p-2}\right) .
$$

Numerical calculations also show that

$$
(p-1)(p-3)(p-5) 3^{p-3} \max \left\{1,2^{p-7}\right\} \leq \frac{(\log 8)^{3}}{196}\left(\frac{7 p}{4 \log p}\right)^{p}
$$

for $6<p \leq 8$. Therefore, from (2.13), we see that (2.2) holds in this case.

For the case where $p>8$, we prove the result by induction. Assume that (2.2) holds for $p-2$. By induction and (2.7), we have

$$
\begin{aligned}
\mathbb{E}|W|^{p-2} & \leq \kappa_{p-2}\left(\sigma^{p-2}+\sigma^{2} \mathbb{E}|T|^{p-4}\right) \\
& \leq \kappa_{p-2}\left(2 \sigma^{p-2}+\mathbb{E}|T|^{p-2}\right) .
\end{aligned}
$$

Combining (2.5) and (2.14), we obtain

$$
\mathbb{E}|W|^{p} \leq(p-1)\left(\frac{2 \kappa_{p-2}}{\theta^{p-3}} \sigma^{p}+\left(\frac{\kappa_{p-2}}{\theta^{p-3}}+\frac{1}{(1-\theta)^{p-3}}\right) \sigma^{2} \mathbb{E}|T|^{p-2}\right) .
$$

The proof is completed if we can choose $0<\theta<1$ such that

$$
\frac{2(p-1) \kappa_{p-2}}{\theta^{p-3}} \leq \kappa_{p} \text { and } \frac{p-1}{(1-\theta)^{p-3}} \leq \frac{\kappa_{p}}{2} .
$$

By Lemma A.1 in the Appendix, we have

$$
\kappa_{p} \geq 8\left(\frac{p-1}{\log (p-1)}\right)^{2} \kappa_{p-2} .
$$

Let

$$
\theta=\theta(p):=\left(\frac{\log ^{2}(p-1)}{4(p-1)}\right)^{1 /(p-3)} .
$$

Then $0<\theta<1$ and the first half of (2.16) holds by (2.17). By Lemma A.2 (in the Appendix), the second half of (2.16) holds.

The proof of the proposition is completed.

We now present a simple proof of the Rosenthal inequality (Rosenthal (1970)) for sums of mean zero independent random variables by using Proposition 2.1. If $\left\{X_{i}, 1 \leq i \leq n\right\}$ are independent symmetric random variables, Johnson, Schechtman and Zinn (1985) proved that

$$
\left\|\sum_{i=1}^{n} X_{i}\right\|_{p} \leq \frac{K p}{\log p} \max \left\{\left\|\sum_{i=1}^{n} X_{i}\right\|_{2},\left(\sum_{i=1}^{n}\left\|X_{i}\right\|_{p}^{p}\right)^{1 / p}\right\} \text { for all } p \geq 2,
$$

where $K$ is a universal constant satisfying $\frac{1}{e \sqrt{2}} \leq K \leq 7.35$. Johnson, Schechtman and Zinn (1985) also proved that the rate $p / \log p$ is optimal. Latała (1997) showed that

imsart-generic ver. 2014/10/16 file: 2020.06.14.Jack_measures.tex date: June 16, 2020 
(2.18) holds with $K$ approximately equal to $2 e$ (see Theorem 2 and Corrolary 3 in Latała (1997)). In Ibragimov and Sharakhmetov (1997), the authors proved that the constant $K$ in (2.18) is approximated 1/e when $p$ large enough (see the Corrolary in page 259 in Ibragimov and Sharakhmetov (1997)). However, we are not aware of any result in the literature (even with assuming the symmetry of the random variables) which proved (2.18) holds with $K \leq 3.5$ for all $p \geq 2$ as given in the following proposition.

Proposition 2.2. Let $p \geq 2$ and $\left\{X_{i}, 1 \leq i \leq n\right\}$ be a collection of $n$ independent mean zero random variables with $\mathbb{E}\left|X_{i}\right|^{p}<\infty, 1 \leq i \leq n$. Then

$$
\left\|\sum_{i=1}^{n} X_{i}\right\|_{p} \leq \frac{3.5 p}{\log p} \max \left\{\left\|\sum_{i=1}^{n} X_{i}\right\|_{2},\left(\sum_{i=1}^{n}\left\|X_{i}\right\|_{p}^{p}\right)^{1 / p}\right\} .
$$

Proof. Let $W=\sum_{i=1}^{n} X_{i}$ and $\sigma^{2}=\operatorname{Var}(W)$. Denote $\operatorname{Var}\left(X_{i}\right)$ by $\sigma_{i}^{2}, 1 \leq i \leq n$. Let $X_{i}^{*}$ have the $X_{i}$-zero biased distribution with $\left\{X_{i}^{*}, 1 \leq i \leq n\right\}$ mutually independent and $X_{i}^{*}$ independent of $\left\{X_{j}, j \neq i\right\}$. Let $I$ be a random index, independent of $\left\{X_{i}, X_{i}^{*}, 1 \leq i \leq n\right\}$, with the distribution

$$
\mathbb{P}(I=i)=\frac{\sigma_{i}^{2}}{\sigma^{2}} .
$$

The argument proving part (v) of Lemma 2.1 in Goldstein and Reinert (1997) shows that removing $X_{I}$ and replacing it by $X_{I}^{*}$ gives a random variable $W^{*}$ with the $W$-zero biased distribution, that is,

$$
W^{*}=W-X_{I}+X_{I}^{*}
$$

has the $W$-zero biased distribution.

Let $\kappa_{p}$ be as in Proposition 2.1. By Proposition 2.1, we have

$$
\begin{aligned}
\mathbb{E}|W|^{p} & \leq \kappa_{p}\left(\sigma^{p}+\sigma^{2} \mathbb{E}\left|W^{*}-W\right|^{p-2}\right) \\
& =\kappa_{p}\left(\sigma^{p}+\sigma^{2} \mathbb{E}\left|X_{I}-X_{I}^{*}\right|^{p-2}\right) \\
& =\kappa_{p}\left(\sigma^{p}+\sigma^{2} \sum_{i=1}^{n} \mathbb{E}\left|X_{i}-X_{i}^{*}\right|^{p-2} \sigma_{i}^{2} / \sigma^{2}\right) \\
& \leq \kappa_{p}\left(\sigma^{p}+\max \left\{1,2^{p-3}\right\} \sum_{i=1}^{n} \sigma_{i}^{2}\left(\mathbb{E}\left|X_{i}\right|^{p-2}+\mathbb{E}\left|X_{i}^{*}\right|^{p-2}\right)\right) \\
& \leq \kappa_{p}\left(\sigma^{p}+2^{p-2} \sum_{i=1}^{n} \sigma_{i}^{2}\left(\mathbb{E}\left|X_{i}\right|^{p-2}+\mathbb{E}\left|X_{i}^{*}\right|^{p-2}\right)\right) .
\end{aligned}
$$

By Hölder's inequality, we have for all $1 \leq i \leq n$,

$$
\sigma_{i}^{2} \mathbb{E}\left|X_{i}\right|^{p-2} \leq\left(\mathbb{E}\left|X_{i}\right|^{p}\right)^{2 / p}\left(\mathbb{E}\left|X_{i}\right|^{p}\right)^{(p-2) / p}=\mathbb{E}\left|X_{i}\right|^{p} .
$$

With the function $f$ as defined in (2.3), it follows from (2.1) that

$$
(p-1) \sigma_{i}^{2} \mathbb{E}\left|X_{i}^{*}\right|^{p-2}=\mathbb{E}\left|X_{i}\right|^{p} .
$$


Combining (2.20)-(2.22), we have

$$
\mathbb{E}|W|^{p} \leq \kappa_{p}\left(\sigma^{p}+2^{p-1} \sum_{i=1}^{n} \mathbb{E}\left|X_{i}\right|^{p}\right) \leq 2^{p} \kappa_{p} \max \left\{\sigma^{p}, \sum_{i=1}^{n} \mathbb{E}\left|X_{i}\right|^{p}\right\},
$$

which proves (2.19).

\section{Uniform and non-uniform Kolmogorov bounds for zero-bias couplings}

Optimal bounds on the Kolmogorov distance for zero-bias couplings have already been obtained by Goldstein (2005) provided the difference between the original random variable and its zero bias transform is properly bounded. In this section, we improved the mentioned result by Goldstein (2005) in two directions: firstly, a truncation argument is used to go beyond boundedness, and secondly, non-uniform bounds with polynomial decay are provided. The following theorem gives the Kolmogorov bound in normal approximation for $W^{*}$.

Theorem 3.1. Let $W$ be such that $\mathbb{E} W=0$ and $\operatorname{Var}(W)=1$, and let $W^{*}$ be $W$ zero biased and be defined on the same probability space as $W$. Let $T=W^{*}-W$.

(i) We have

$$
\sup _{x \in \mathbb{R}}\left|\mathbb{P}\left(W^{*} \leq x\right)-\Phi(x)\right| \leq\left(1+\frac{\sqrt{2 \pi}}{4}\right) \sqrt{\mathbb{E} T^{2}} .
$$

(ii) Let $p \geq 2$. Then for all $x \in \mathbb{R}$,

$$
\left|\mathbb{P}\left(W^{*} \leq x\right)-\Phi(x)\right| \leq \frac{C_{p}}{1+|x|^{p}}\left(\frac{p}{\log p}\right)^{p}\left(\sqrt{\mathbb{E} T^{2}}+\sqrt{\mathbb{E}|T|^{2 p+2}}\right) .
$$

Proof. For $x \in \mathbb{R}$, let $f_{x}$ be the unique bounded solution of the Stein equation

$$
f^{\prime}(w)-w f(w)=1(w \leq x)-\Phi(x),
$$

and let

$$
g_{x}(w)=\left(w f_{x}(w)\right)^{\prime} .
$$

We have $0<f_{x}(w) \leq \sqrt{2 \pi} / 4$ and $\left|f_{x}^{\prime}(w)\right| \leq 1$ for all $w \in \mathbb{R}$ (see Stein (1986)). Therefore

$$
\begin{gathered}
\left|g_{x}(w)\right|=\left|f_{x}(w)+w f_{x}^{\prime}(w)\right| \leq 1+|w| \text { for all } w \in \mathbb{R}, \\
\mathbb{E}\left|T f_{x}(W+T)\right| \leq \frac{\sqrt{2 \pi}}{4} \mathbb{E}|T| \leq \frac{\sqrt{2 \pi}}{4} \sqrt{\mathbb{E} T^{2}},
\end{gathered}
$$

and

$$
\mathbb{E} \mid\left(W\left(f_{x}(W+T)-f_{x}(W)\right)|\leq \mathbb{E}| W T \mid \leq \sqrt{\mathbb{E} W^{2} \mathbb{E} T^{2}}=\sqrt{\mathbb{E} T^{2}} .\right.
$$


Since

$$
\begin{aligned}
\left|\mathbb{P}\left(W^{*} \leq x\right)-\Phi(x)\right| & =\left|\mathbb{E} f_{x}^{\prime}\left(W^{*}\right)-\mathbb{E} W^{*} f_{x}\left(W^{*}\right)\right| \\
& =\left|\mathbb{E} W f_{x}(W)-\mathbb{E}(W+T) f_{x}(W+T)\right| \\
& \leq \mathbb{E} \mid\left(W\left(f_{x}(W+T)-f_{x}(W)\right)|+\mathbb{E}| T f_{x}(W+T) \mid,\right.
\end{aligned}
$$

the conclusion (3.1) follows by combining (3.6), (3.7), and (3.8). Theorem 3.1(i) is proved.

To prove Theorem 3.1(ii), it suffices to consider the case where $x \geq 0$ since we can simply apply the result to $-W^{*}$ when $x<0$ (see (2.59) in Chen, Goldstein and Shao (2011)). In view of the uniform bound (3.1), it suffices to consider the case where $x \geq 2$. By applying Markov's inequality and Proposition 2.1, we have

$$
\begin{aligned}
\left|P\left(W^{*} \leq x\right)-\Phi(x)\right| & \leq \max \left\{P\left(W^{*}>x\right), 1-\Phi(x)\right\} \\
& \leq \max \left\{\frac{\mathbb{E}\left|W^{*}\right|^{p+1}}{x^{p+1}}, 1-\Phi(x)\right\} \\
& =\max \left\{\frac{\mathbb{E}|W|^{p+3}}{(p+2) x^{p+1}}, 1-\Phi(x)\right\} \\
& \leq \max \left\{\frac{\kappa_{p+3}\left(1+\mathbb{E}|T|^{p+1}\right)}{(p+2) x^{p+1}}, 1-\Phi(x)\right\} .
\end{aligned}
$$

By using the fact that $\sqrt{2 \pi}(1-\Phi(x)) \leq e^{-x^{2} / 2} / x$ for all $x>0$, we have

$$
\max _{x>0} x^{p+1}(1-\Phi(x)) \leq \frac{1}{\sqrt{2 \pi}} \max _{x>0} x^{p} e^{-x^{2} / 2}=\frac{1}{\sqrt{2 \pi}}\left(\frac{\sqrt{p}}{\sqrt{e}}\right)^{p} .
$$

Combining (3.9) and (3.10), we obtain

$$
\left|P\left(W^{*} \leq x\right)-\Phi(x)\right| \leq \frac{C_{p}}{1+x^{p}}\left(\frac{p}{\log p}\right)^{p}\left(1+\sqrt{\mathbb{E}|T|^{2 p+2}}\right) .
$$

If $\mathbb{E}|T|^{2 p+2} \geq 1$, then $1+\sqrt{\mathbb{E}|T|^{2 p+2}} \leq 2 \sqrt{\mathbb{E}|T|^{2 p+2}}$. Therefore (3.2) holds by (3.11). It remains to consider the case where $\mathbb{E}|T|^{2 p+2}<1$. In this case, by applying Proposition 2.1 and Jensen's inequality, we have

$$
\begin{aligned}
\mathbb{E}|W|^{2 p} & \leq \kappa_{2 p}\left(1+\mathbb{E}|T|^{2 p-2}\right) \\
& \leq 2 \kappa_{2 p} \leq C_{p}\left(\frac{p}{\log p}\right)^{2 p},
\end{aligned}
$$

and

$$
\begin{aligned}
\mathbb{E}|W|^{2 p+2} & \leq \kappa_{2 p+2}\left(1+\mathbb{E}|T|^{2 p}\right) \\
& \leq 2 \kappa_{2 p+2} \leq C_{p}\left(\frac{p}{\log p}\right)^{2 p} .
\end{aligned}
$$


Since

$$
\begin{aligned}
\mathbb{P}\left(W^{*} \leq x\right)-\Phi(x) & =\mathbb{E}\left\{W f_{x}(W)-W^{*} f_{x}\left(W^{*}\right)\right\} \\
& =-\mathbb{E} \int_{0}^{T} g_{x}(W+t) \mathrm{d} t
\end{aligned}
$$

we have

$$
\left|\mathbb{P}\left(W^{*} \leq x\right)-\Phi(x)\right| \leq R_{1}+R_{2},
$$

where

$$
R_{1}=\left|\mathbb{E} \int_{0}^{T} g_{x}(W+t)\left(\mathbf{1}(W+t \leq 0)+\mathbf{1}\left(0<W+t \leq \frac{x}{2}\right)\right) \mathrm{d} t\right|
$$

and

$$
R_{2}=\left|\mathbb{E} \int_{0}^{T} g_{x}(W+t) \mathbf{1}\left(W+t>\frac{x}{2}\right) \mathrm{d} t\right| .
$$

From the definition of $f_{x}$ and $g_{x}$, we have (see Chen and Shao (2001))

$$
g_{x}(w)= \begin{cases}\left(\sqrt{2 \pi}\left(1+w^{2}\right) e^{w^{2} / 2}(1-\Phi(w))-w\right) \Phi(x) & \text { if } w \geq x, \\ \left.\sqrt{2 \pi}\left(1+w^{2}\right) e^{w^{2} / 2} \Phi(w)+w\right)(1-\Phi(x)) & \text { if } w<x .\end{cases}
$$

Chen and Shao (2001) proved that $g_{x} \geq 0, g_{x}(w) \leq 2(1-\Phi(x))$ for $w \leq 0$, and $g_{x}$ is increasing for $0 \leq w<x$. From $(3.17)$ and the fact that $\sqrt{2 \pi}(1-\Phi(x)) \leq$ $e^{-x^{2} / 2} / x$ for all $x>0$, we have

$$
\begin{aligned}
g_{x}(x / 2) & =\left(\sqrt{2 \pi}\left(1+\frac{x^{2}}{4}\right) e^{x^{2} / 8} \Phi(x / 2)+\frac{x}{2}\right)(1-\Phi(x)) \\
& \leq\left(\frac{1}{x}+\frac{x}{4}\right) e^{-3 x^{2} / 8}+\frac{1}{2 \sqrt{2 \pi}} e^{-x^{2} / 2} .
\end{aligned}
$$

For all $r \geq 1$, a straightforward calculation shows that

$$
\max _{x>0} x^{r} e^{-x^{2} / 2}<\max _{x>0} x^{r} e^{-3 x^{2} / 8}=\left(\frac{2 \sqrt{r}}{\sqrt{3 e}}\right)^{r} .
$$

Therefore, from (3.15) and (3.18), we have

$$
\begin{aligned}
R_{1} & \leq \mathbb{E} \int_{0}^{|T|}\left(2(1-\Phi(x))+g_{x}(x / 2)\right) \mathrm{d} t \\
& \leq \frac{C_{p}}{1+x^{p}}\left(\frac{p}{\log p}\right)^{p} \mathbb{E}|T| \leq \frac{C_{p}}{1+x^{p}}\left(\frac{p}{\log p}\right)^{p} \sqrt{\mathbb{E} T^{2}} .
\end{aligned}
$$

To bound $R_{2}$, we estimate

$$
\mathbf{1}\left(W+t>\frac{x}{2}\right) \leq \frac{C_{p}}{1+x^{p}}\left(|W|^{p}+|T|^{p}\right) \text { for all } 0 \leq t \leq|T| .
$$


Combining (3.5) and (3.20), we have

$$
\begin{aligned}
R_{2} & \leq \frac{C_{p}}{1+x^{p}} \mathbb{E} \int_{0}^{|T|}(1+|W|+|T|)\left(|W|^{p}+|T|^{p}\right) \mathrm{d} t \\
& =\frac{C_{p}}{1+x^{p}} \mathbb{E}(1+|W|+|T|)\left(|W|^{p}|T|+|T|^{p+1}\right) .
\end{aligned}
$$

We bound each term in (3.21) as follows. Firstly, we have

$$
\mathbb{E}|T|^{p+1} \leq \sqrt{\mathbb{E} T^{2 p+2}} \text { and } \mathbb{E}|W||T|^{p+1} \leq \sqrt{\mathbb{E} W^{2} \mathbb{E} T^{2 p+2}}=\sqrt{\mathbb{E} T^{2 p+2}} .
$$

Secondly, by using the CauchySchwarz inequality, (3.12) and (3.13), and by noting that $\mathbb{E}|T|^{2 p+2}<1$, we have

$$
\begin{aligned}
& \mathbb{E}|W|^{p}|T| \leq \sqrt{\mathbb{E}|W|^{2 p} \mathbb{E} T^{2}} \leq C_{p}\left(\frac{p}{\log p}\right)^{p} \sqrt{\mathbb{E} T^{2}}, \\
& \mathbb{E}|W|^{p+1}|T| \leq \sqrt{\mathbb{E}|W|^{2 p+2} \mathbb{E} T^{2}} \leq C_{p}\left(\frac{p}{\log p}\right)^{p} \sqrt{\mathbb{E} T^{2}},
\end{aligned}
$$

and

$$
\mathbb{E}|T|^{p+2} \leq \sqrt{\mathbb{E} T^{2} \mathbb{E} T^{2 p+2}} \leq \sqrt{\mathbb{E} T^{2}} .
$$

Finally,

$$
\begin{aligned}
\mathbb{E}|W|^{p}|T|^{2} & =\mathbb{E}\left(\left(|W|^{p+1}|T|\right)^{p /(p+1)}\left(|T|^{p+2}\right)^{1 /(p+1)}\right) \\
& \leq \mathbb{E}\left(|W|^{p+1}|T|+|T|^{p+2}\right) \\
& \leq C_{p}\left(\frac{p}{\log p}\right)^{p} \sqrt{\mathbb{E} T^{2}}
\end{aligned}
$$

where we have used (2.6) in the first inequality, and (3.24) and (3.25) in the second inequality. From (3.21)-(3.26), we have

$$
R_{2} \leq \frac{C_{p}}{1+x^{p}}\left(\frac{p}{\log p}\right)^{p}\left(\sqrt{\mathbb{E} T^{2}}+\sqrt{\mathbb{E}|T|^{2 p+2}}\right) .
$$

Combining (3.14), (3.19) and (3.27), we obtain (3.2).

Theorem 3.1 is a normal approximation for $W^{*}$. When $T=W^{*}-W$ has fast decaying tails, by using Theorem 3.1, we can obtain useful bounds in normal approximation for $W$. This gives us the following theorem.

Theorem 3.2. Let $W$ be such that $\mathbb{E} W=0$ and $\operatorname{Var}(W)=1$, and let $W^{*}$ be $W$-zero biased and defined on the same probability space as $W$. Let $T=W^{*}-W$ and $\varepsilon>0$ be arbitrary.

(i) We have

$$
\sup _{x \in \mathbb{R}}|\mathbb{P}(W \leq x)-\Phi(x)| \leq\left(1+\frac{\sqrt{2 \pi}}{4}\right) \sqrt{\mathbb{E} T^{2}}+\frac{\varepsilon}{\sqrt{2 \pi}}+\mathbb{P}(|T|>\varepsilon) .
$$


(ii) Let $p \geq 2$. Then for all $x \in \mathbb{R}$,

$$
\begin{aligned}
& |\mathbb{P}(W \leq x)-\Phi(x)| \\
& \leq \frac{C_{p}}{1+|x|^{p}}\left(\frac{p}{\log p}\right)^{p}\left(\sqrt{\mathbb{E} T^{2}}+\sqrt{\mathbb{E}|T|^{2 p+2}}+\varepsilon+\sqrt{\mathbb{P}(|T|>\varepsilon)}\right) .
\end{aligned}
$$

Remark 3.3. If $|T| \leq \varepsilon$ almost surely, then (3.28) reduces to

$$
\sup _{x \in \mathbb{R}}|\mathbb{P}(W \leq x)-\Phi(x)| \leq\left(1+\frac{\sqrt{2 \pi}}{4}+\frac{1}{\sqrt{2 \pi}}\right) \varepsilon .
$$

In Theorem 1.1 in Goldstein (2005), the author considered the following distance between $W$ and the standard normal random variable $Z$

$$
\mathrm{d}(W, Z)=\sup _{h \in \mathcal{H}}|\mathbb{E} h(W)-\mathbb{E} h(Z)|,
$$

where $\mathcal{H}$ is a class of measurable functions on the real line which contains the collection of indicators of all half lines. When $\mathcal{H}$ coincides with the collection of indicators of all half lines, the author proved that (see the first half of (10) in Goldstein (2005))

$$
\sup _{x \in \mathbb{R}}|\mathbb{P}(W \leq x)-\Phi(x)| \leq(127+12 \varepsilon) \varepsilon .
$$

Proof of Theorem 3.2. Let $\varepsilon>0$ be arbitrary. Then by (3.1), we have

$$
\begin{aligned}
\mathbb{P}(W \leq x)-\Phi(x)= & \mathbb{P}\left(W^{*} \leq x+W^{*}-W\right)-\Phi(x) \\
\leq & \mathbb{P}\left(W^{*} \leq x+\varepsilon\right)-\Phi(x+\varepsilon)+\Phi(x+\varepsilon)-\Phi(x) \\
& \quad+\mathbb{P}\left(W^{*}-W>\varepsilon\right) \\
\leq & \left(1+\frac{\sqrt{2 \pi}}{4}\right) \sqrt{\mathbb{E} T^{2}}+\frac{\varepsilon}{\sqrt{2 \pi}}+\mathbb{P}\left(\left|W^{*}-W\right|>\varepsilon\right),
\end{aligned}
$$

and

$$
\begin{aligned}
\mathbb{P}(W \leq x)-\Phi(x) \geq & \mathbb{P}\left(W^{*} \leq x-\varepsilon\right)-\Phi(x-\varepsilon)+\Phi(x-\varepsilon)-\Phi(x) \\
& -\mathbb{P}\left(W^{*}-W<-\varepsilon\right) \\
\geq & \left(1+\frac{\sqrt{2 \pi}}{4}\right) \sqrt{\mathbb{E} T^{2}}-\frac{\varepsilon}{\sqrt{2 \pi}}-\mathbb{P}\left(\left|W^{*}-W\right|>\varepsilon\right) .
\end{aligned}
$$

Combining (3.32) and (3.33), we obtain (3.28).

To prove (3.29), it suffices to consider $x \geq 2$, as in the proof of (3.2). Similar to the proof of (3.11), we have

$$
|P(W \leq x)-\Phi(x)| \leq \frac{C_{p}}{1+x^{p}}\left(\frac{p}{\log p}\right)^{p}\left(1+\sqrt{\mathbb{E}|T|^{2 p+2}}\right) .
$$


Therefore, if either $\mathbb{E}|T|^{2 p+2} \geq 1$ or $\varepsilon \geq 1$, then (3.29) holds. It remains to consider the case where $\mathbb{E}|T|^{2 p+2}<1$ and $\varepsilon<1$. In this case, similar to (3.12), we have

$$
\mathbb{E}\left|W^{*}\right|^{2 p}=\frac{\mathbb{E}|W|^{2 p+2}}{(2 p+1) \mathbb{E} W^{2}} \leq \frac{2 \kappa_{2 p+2}}{2 p+1} \leq C_{p}\left(\frac{p}{\log p}\right)^{2 p} .
$$

Since

$$
\begin{aligned}
\mathbb{P}\left(W^{*}>x+\varepsilon\right) & =\mathbb{P}\left(W^{*}>x+\varepsilon, T>\varepsilon\right)+\mathbb{P}\left(W^{*}>x+\varepsilon, T \leq \varepsilon\right) \\
& \leq \mathbb{P}\left(W^{*}>x, T>\varepsilon\right)+\mathbb{P}(W>x),
\end{aligned}
$$

we have

$$
\begin{aligned}
\mathbb{P}(W \leq x)-\Phi(x) & =1-\mathbb{P}(W>x)-\Phi(x) \\
& \leq 1-\mathbb{P}\left(W^{*}>x+\varepsilon\right)-\Phi(x)+\mathbb{P}\left(W^{*}>x, T>\varepsilon\right) .
\end{aligned}
$$

Combining (3.2), (3.35) and (3.36), we have

$$
\begin{aligned}
\mathbb{P} & (W \leq x)-\Phi(x) \leq \mathbb{P}\left(W^{*} \leq x+\varepsilon\right)-\Phi(x+\varepsilon) \\
& +\Phi(x+\varepsilon)-\Phi(x)+\mathbb{P}\left(W^{*}>x, T>\varepsilon\right) \\
\leq & \frac{C_{p}}{1+x^{p}}\left(\frac{p}{\log p}\right)^{p}\left(\sqrt{\mathbb{E} T^{2}}+\sqrt{\mathbb{E}|T|^{2 p+2}}\right) \\
& +\frac{\varepsilon e^{-x^{2} / 2}}{\sqrt{2 \pi}}+\sqrt{\mathbb{P}(|T|>\varepsilon)} \sqrt{\mathbb{P}\left(\left|W^{*}\right|>x\right)} \\
\leq & \frac{C_{p}}{1+x^{p}}\left(\frac{p}{\log p}\right)^{p}\left(\sqrt{\mathbb{E} T^{2}}+\sqrt{\mathbb{E}|T|^{2 p+2}}+\varepsilon\right) \\
& +\frac{\sqrt{\mathbb{P}(|T|>\varepsilon)} \sqrt{\mathbb{E}\left|W^{*}\right|^{2 p}}}{x^{p}} \\
\leq & \frac{C_{p}}{1+x^{p}}\left(\frac{p}{\log p}\right)^{p}\left(\sqrt{\mathbb{E} T^{2}}+\sqrt{\mathbb{E}|T|^{2 p+2}}+\varepsilon+\sqrt{\mathbb{P}(|T|>\varepsilon)}\right) .
\end{aligned}
$$

Similarly, by noting that $x-\varepsilon>x-1 \geq 1$, we can show that

$$
\begin{aligned}
& \mathbb{P}(W \leq x)-\Phi(x) \\
& \geq-\frac{C_{p}}{1+x^{p}}\left(\frac{p}{\log p}\right)^{p}\left(\sqrt{\mathbb{E} T^{2}}+\sqrt{\mathbb{E}|T|^{2 p+2}}+\varepsilon+\sqrt{\mathbb{P}(|T|>\varepsilon)}\right) .
\end{aligned}
$$

Combining (3.37) and (3.38), we obtain (3.29).

\section{Proofs of the main results}

The rate in the following proposition is better than that of Theorem 1.1 in the case where $\alpha \geq n^{1+\delta}$ for some $\delta>0$ fixed. We would like to note here that when $1 \leq \alpha \leq n / \log ^{2} n$ or $\alpha \geq n^{1+\delta}$ for some $\delta>0$ fixed, the convergence rate obtained in Proposition 4.1 is exactly the rate in Fulman's conjecture. Chen, Goldstein and Röllin (2020) also obtained the bound $O(\sqrt{\alpha} / n)$ for the case $\alpha \geq n^{1+\delta}$ by applying induction with Stein's method. 
Proposition 4.1. Let $n \geq 3$ be an integer. Let $\alpha \geq 1$ and $W_{n, \alpha}$ be as in (1.5). Then

$$
\sup _{x \in \mathbb{R}}\left|\mathbb{P}_{\alpha}\left(W_{n, \alpha} \leq x\right)-\Phi(x)\right| \leq 8.2 \max \left\{\frac{1}{\sqrt{n}}, \frac{\sqrt{\alpha} \log n}{n}\right\} .
$$

If, in addition, $\alpha \geq n^{1+\delta}$ for some $\delta:=\delta(\alpha, n)>0$, then

$$
\sup _{x \in \mathbb{R}}\left|\mathbb{P}_{\alpha}\left(W_{n, \alpha} \leq x\right)-\Phi(x)\right| \leq\left(4.7+\frac{3.1}{\delta}\right) \frac{\sqrt{\alpha}}{n} .
$$

Remark 4.2. If $\alpha>n$, then we can write $\alpha=n^{1+\delta}$, where

$$
\delta=\frac{\log \alpha-\log n}{\log n}>0 .
$$

Applying (4.2), we have

$$
\sup _{x \in \mathbb{R}}\left|\mathbb{P}_{\alpha}\left(W_{n, \alpha} \leq x\right)-\Phi(x)\right| \leq \frac{4.7 \log \alpha-1.6 \log n}{\log \alpha-\log n} \frac{\sqrt{\alpha}}{n} .
$$

We make some notes as follows.

(i) If $\alpha \sim K n$ for some $K>1$ fixed, then the rate obtained in (4.3) is $O\left(\frac{\sqrt{\alpha} \log n}{n}\right)$ which is the same as the rate obtained in (4.1).

(ii) If $\alpha \sim n(\log n)^{K}$ for some $K>0$ fixed, then the rate obtained in (4.3) is $O\left(\frac{\sqrt{\alpha} \log n}{n \log (\log n)}\right)$ which is better than the rate obtained in (4.1).

(iii) If $\alpha \geq n^{1+\delta}$ for some $\delta>0$ fixed, then the convergence rate obtained in (4.3) is $O\left(\frac{\sqrt{\alpha}}{n}\right)$ which is exactly the rate in Fulman's conjecture.

We will prove Proposition 4.1 by applying Theorem 3.2. In Kerov (2000), the author proved that there is a growth process giving a sequence of partitions $(\lambda(1), \ldots, \lambda(n))$ with $\lambda(j)$ distributed according to the $\mathrm{Jack}_{\alpha}$ measure on partitions of size $j$. We refer to Fulman (2004) for details. Given Kerov's process, let $X_{1, \alpha}=0, X_{j, \alpha}=c_{\alpha}(a)$ where $a$ is the box added to $\lambda(j-1)$ to obtain $\lambda(j)$ and the " $\alpha$-content" $c_{\alpha}(a)$ of a box $a$ is defined to be $\alpha$ (column number of $a-$ 1 ) - (row number of $a-1), j \geq 2$. Then one can write (see Fulman (2006); Fulman and Goldstein (2011))

$$
W_{n, \alpha}=\frac{\sum_{j=1}^{n} X_{j, \alpha}}{\sqrt{\alpha\left(\begin{array}{c}
n \\
2
\end{array}\right)}} .
$$

Therefore, constructing $\nu$ from the $\mathrm{Jack}_{\alpha}$ measure on partitions of $n-1$ and then taking one step in Kerov's growth process yields $\lambda$ with the $\mathrm{Jack}_{\alpha}$ measure on partitions of $n$, we have

$$
W_{n, \alpha}=V_{n, \alpha}+\eta_{n, \alpha},
$$


where

$$
V_{n, \alpha}=\frac{\sum_{x \in \nu} c_{\alpha}(x)}{\sqrt{\alpha\left(\begin{array}{c}
n \\
2
\end{array}\right)}}=\sqrt{\frac{n-2}{n}} W_{n-1, \alpha}, \eta_{n, \alpha}=\frac{X_{n, \alpha}}{\sqrt{\alpha\left(\begin{array}{c}
n \\
2
\end{array}\right)}}=\frac{c_{\alpha}(\lambda / \nu)}{\sqrt{\alpha\left(\begin{array}{c}
n \\
2
\end{array}\right)}},
$$

and $c_{\alpha}(\lambda / \nu)$ denotes the $\alpha$-content of the box added to $\nu$ to obtain $\lambda$. Fulman (2006) proved that

$$
\begin{gathered}
E W_{n, \alpha}=0, E W_{n, \alpha}^{2}=1, \\
E \eta_{n, \alpha}=0, E \eta_{n, \alpha}^{2}=\frac{2}{n}
\end{gathered}
$$

and

$$
E \eta_{n, \alpha}^{4}=\frac{2}{n^{2}}\left(\frac{4 n-6}{n-1}+\frac{2(\alpha-1)^{2}}{\alpha(n-1)}\right) .
$$

From Theorems 3.1 and 4.1 in Fulman and Goldstein (2011), there exists a random variable $\eta_{n, \alpha}^{*}$ defined on the same probability space with $\eta_{n, \alpha}$, and satisfying that $\eta_{n, \alpha}^{*}$ has $\eta_{n, \alpha}$-zero biased distribution and that

$$
W_{n, \alpha}^{*}=V_{n, \alpha}+\eta_{n, \alpha}^{*}
$$

has $W_{n, \alpha}$-zero biased distribution. Here and thereafter, we denote

$$
T_{n, \alpha}=\eta_{n, \alpha}-\eta_{n, \alpha}^{*} .
$$

The following lemma gives a bound for $\mathbb{E}\left(\eta_{n, \alpha}^{*}\right)^{2}$.

Lemma 4.3. For $\alpha \geq 1$, we have

$$
\begin{aligned}
\mathbb{E}\left(\eta_{n, \alpha}^{*}\right)^{2} & =\frac{1}{3 n}\left(\frac{4 n-6}{n-1}+\frac{2(\alpha-1)^{2}}{\alpha(n-1)}\right) \\
& \leq \frac{1}{3 n}\left(4+\frac{2 \alpha}{n-1}\right) .
\end{aligned}
$$

Proof. Applying (2.1) with $f(x)=x^{3}$, we have

$$
\mathbb{E}\left(\eta_{n, \alpha}^{*}\right)^{2}=\frac{\mathbb{E}\left(\eta_{n, \alpha}\right)^{4}}{3 \mathbb{E} \eta_{n, \alpha}^{2}} .
$$

Combining (4.8), (4.9) and (4.12), we obtain (4.11).

For a partition $\lambda$ of a positive integer $n$, we recall that the length of row $i$ of $\lambda$ and the length of column $i$ of $\lambda$ are denoted by $\lambda_{i}$ and $\lambda_{i}^{\prime}$, respectively.

From a computation in the proof of Lemma 6.6 in Fulman (2004) and Stirling's formula, we have the following lemma.

Lemma 4.4. Let $\alpha>0$. Then for $1 \leq l \leq n$, we have

$$
\mathbb{P}_{\alpha}\left(\lambda_{1}=l\right) \leq \frac{\alpha}{2 \pi}\left(\frac{n e^{2}}{\alpha l^{2}}\right)^{l} .
$$


Proof. It is proved by Fulman (2004) that

$$
\mathbb{P}_{\alpha}\left(\lambda_{1}=l\right) \leq\left(\frac{n}{\alpha}\right)^{l} \frac{\alpha l}{(l !)^{2}}
$$

By Stirling's formula, we have for all $l \geq 1$,

$$
l ! \geq \sqrt{2 \pi l}\left(\frac{l}{e}\right)^{l}
$$

Combining (4.14) and (4.15), we have (4.13).

In order to apply Theorem 3.2 , we need to bound $\mathbb{P}\left(\left|T_{n, \alpha}\right|>\varepsilon\right)$ for suitably chosen $\varepsilon$. The following lemma shows that $\left|T_{n, \alpha}\right|$ has a very light tail.

Lemma 4.5. For all $\alpha \geq 1$ and $q>1$, we have

$$
\mathbb{P}_{\alpha}\left(\left|T_{n, \alpha}\right|>\frac{2 e \sqrt{2 q}}{\sqrt{n-1}}\right) \leq \frac{\alpha}{\pi(q-1) q^{e \sqrt{q n / \alpha}}}+\frac{\alpha^{2} q(e \sqrt{q n / \alpha}(q-1)+q+1)}{\pi(n-1)(q-1)^{3} q^{e \sqrt{q n / \alpha}}}
$$

Proof. First, we take an arbitrary $\alpha>0$. It follows from (4.13) that

$$
\mathbb{P}_{\alpha}\left(\lambda_{1}=k+1\right) \leq \frac{\alpha}{2 \pi q^{k+1}}
$$

for all $k \geq e \sqrt{q n / \alpha}$. Therefore

$$
\begin{aligned}
\mathbb{P}_{\alpha}\left(\lambda_{1}-1>e \sqrt{q n / \alpha}\right) & =\mathbb{P}_{\alpha}\left(\lambda_{1}-1 \geq\lfloor e \sqrt{q n / \alpha}\rfloor+1\right) \\
& =\sum_{k \geq\lfloor e \sqrt{q n / \alpha}\rfloor+1} \mathbb{P}_{\alpha}\left(\lambda_{1}=k+1\right) \\
& \leq \frac{\alpha}{2 \pi} \sum_{k \geq\lfloor e \sqrt{q n / \alpha}\rfloor+1} \frac{1}{q^{k+1}} \\
& =\frac{q \alpha}{2 \pi(q-1) q^{\lfloor e \sqrt{q n / \alpha}\rfloor+2}} \\
& \leq \frac{\alpha}{2 \pi(q-1) q^{e \sqrt{q n / \alpha}}} .
\end{aligned}
$$

We note that from the definition of Jack measure, $\mathbb{P}_{\alpha}(\lambda)=\mathbb{P}_{1 / \alpha}\left(\lambda^{t}\right)$, where $\lambda^{t}$ is the transpose partition of $\lambda$. Applying (4.17) with $\alpha$ replaced by $1 / \alpha$, we have

$$
\mathbb{P}_{\alpha}\left(\lambda_{1}^{\prime}-1>e \sqrt{q \alpha n}\right) \leq \frac{1}{2 \pi \alpha(q-1) q^{e \sqrt{q \alpha n}}}
$$


Since $\left|X_{n, \alpha}\right| \leq \max \left\{\alpha\left(\lambda_{1}-1\right), \lambda_{1}^{\prime}-1\right\}$, it follows from (4.17) and (4.18) that

$$
\begin{aligned}
\mathbb{P}_{\alpha}\left(\left|\eta_{n, \alpha}\right|>\frac{e \sqrt{2 q}}{\sqrt{n-1}}\right) & =\mathbb{P}_{\alpha}\left(\frac{\sqrt{2}\left|X_{n, \alpha}\right|}{\sqrt{\alpha n(n-1)}}>\frac{e \sqrt{2 q}}{\sqrt{n-1}}\right) \\
& \leq \mathbb{P}_{\alpha}\left(\max \left\{\alpha\left(\lambda_{1}-1\right), \lambda_{1}^{\prime}-1\right\}>e \sqrt{q \alpha n}\right) \\
& \leq \mathbb{P}_{\alpha}\left(\lambda_{1}-1>e \sqrt{q n / \alpha}\right)+\mathbb{P}_{\alpha}\left(\lambda_{1}^{\prime}-1>e \sqrt{q \alpha n}\right) \\
& \leq \frac{\alpha}{2 \pi(q-1) q^{e \sqrt{q n / \alpha}}}+\frac{1}{2 \pi \alpha(q-1) q^{e \sqrt{q \alpha n}}} .
\end{aligned}
$$

For $\alpha \geq 1$, it reduces to

$$
\mathbb{P}_{\alpha}\left(\left|\eta_{n, \alpha}\right|>\frac{e \sqrt{2 q}}{\sqrt{n-1}}\right) \leq \frac{\alpha}{\pi(q-1) q^{e \sqrt{q n / \alpha}}} .
$$

Recall that if $X$ is a random variable with $\mathbb{E} X=0, \mathbb{E} X^{2}=\sigma^{2}$ and if $X^{*}$ has $X$-zero-biased distribution, then for $x>0$, applying (2.1) with $f_{x}(w)=$ $(w-x) \mathbf{1}(w>x)$, we have

$$
\mathbb{P}\left(X^{*}>x\right)=\mathbb{E}[X(X-x) \mathbf{1}(X>x)] / \sigma^{2} .
$$

By using (4.21) and (4.16), and noting that

$$
\eta_{n, \alpha} \leq \frac{\sqrt{2 \alpha}\left(\lambda_{1}-1\right)}{\sqrt{n(n-1)}}
$$

we have

$$
\begin{aligned}
\mathbb{P}_{\alpha} & \left(\eta_{n, \alpha}^{*}>\frac{e \sqrt{2 q}}{\sqrt{n-1}}\right) \\
& =\frac{n}{2} \mathbb{E}\left(\eta_{n, \alpha}\left(\eta_{n, \alpha}-\frac{e \sqrt{2 q}}{\sqrt{n-1}}\right) \mathbf{1}\left(\eta_{n, \alpha}>\frac{e \sqrt{2 q}}{\sqrt{n-1}}\right)\right) \\
& \leq \frac{\alpha}{n-1} \mathbb{E}\left(\left(\lambda_{1}-1\right)\left(\lambda_{1}-1-e \sqrt{q n / \alpha}\right) \mathbf{1}\left(\lambda_{1}-1>e \sqrt{q n / \alpha}\right)\right) \\
& \leq \frac{\alpha}{n-1} \sum_{k=1}^{\infty} k(k+\lfloor e \sqrt{q n / \alpha}\rfloor) \mathbb{P}_{\alpha}\left(\lambda_{1}=k+\lfloor e \sqrt{q n / \alpha}\rfloor+1\right) \\
& \leq \frac{\alpha^{2}}{2 \pi(n-1)} \sum_{k=1}^{\infty} \frac{k(k+\lfloor e \sqrt{q n / \alpha}\rfloor)}{q^{k+\lfloor e \sqrt{q n / \alpha}\rfloor+1}} \\
& =\frac{\alpha^{2}(\lfloor e \sqrt{q n / \alpha}\rfloor(q-1)+q+1)}{2 \pi(n-1)(q-1)^{3} q^{\lfloor e \sqrt{q n / \alpha}\rfloor}} \\
& \leq \frac{\alpha^{2} q(e \sqrt{q n / \alpha}(q-1)+q+1)}{2 \pi(n-1)(q-1)^{3} q^{e \sqrt{q n / \alpha}}} .
\end{aligned}
$$


Applying (4.13) again, we have

$$
\mathbb{P}_{\alpha}\left(\lambda_{1}^{\prime}=k+1\right)=\mathbb{P}_{1 / \alpha}\left(\lambda_{1}=k+1\right) \leq \frac{1}{2 \pi \alpha q^{k+1}}
$$

for all $k \geq e \sqrt{q \alpha n}$. By using (4.23) and noting that

$$
\eta_{n, \alpha} \geq-\frac{\sqrt{2}\left(\lambda_{1}^{\prime}-1\right)}{\sqrt{\alpha n(n-1)}}
$$

we have

$$
\begin{aligned}
& \mathbb{P}_{\alpha}\left(-\eta_{n, \alpha}^{*}>\frac{e \sqrt{2 q}}{\sqrt{n-1}}\right) \\
& =\frac{n}{2} \mathbb{E}\left(-\eta_{n, \alpha}\left(-\eta_{n, \alpha}-\frac{e \sqrt{2 q}}{n-1}\right) \mathbf{1}\left(-\eta_{n, \alpha}>\frac{e \sqrt{2 q}}{\sqrt{n-1}}\right)\right] \\
& \leq \frac{1}{\alpha(n-1)} \mathbb{E}\left(\left(\lambda_{1}^{\prime}-1\right)\left(\lambda_{1}^{\prime}-1-e \sqrt{q \alpha n}\right) \mathbf{1}\left(\lambda_{1}^{\prime}-1>e \sqrt{q \alpha n}\right)\right) \\
& \leq \frac{1}{\alpha(n-1)} \sum_{k=1}^{\infty} k(k+\lfloor e \sqrt{q \alpha n}\rfloor) \mathbb{P}_{\alpha}\left(\lambda_{1}^{\prime}=k+\lfloor e \sqrt{q \alpha n}\rfloor+1\right) \\
& \leq \frac{1}{2 \pi \alpha^{2}(n-1)} \sum_{k=1}^{\infty} \frac{k(k+\lfloor e \sqrt{q \alpha n}\rfloor)}{q^{k+\lfloor e \sqrt{q \alpha n}\rfloor+1}} \\
& =\frac{\lfloor e \sqrt{q \alpha n}\rfloor(q-1)+q+1}{2 \pi \alpha^{2}(n-1)(q-1)^{3} q^{\lfloor e \sqrt{q \alpha n}\rfloor}} \\
& \leq \frac{q(e \sqrt{q \alpha n}(q-1)+q+1)}{2 \pi \alpha^{2}(n-1)(q-1)^{3} q^{e \sqrt{q \alpha n}} .}
\end{aligned}
$$

For $\alpha \geq 1,(4.22)$ and (4.24) reduce to

$$
\mathbb{P}_{\alpha}\left(\left|\eta_{n, \alpha}^{*}\right|>\frac{e \sqrt{2 q}}{\sqrt{n-1}}\right) \leq \frac{\alpha^{2} q(e \sqrt{q n / \alpha}(q-1)+q+1)}{\pi(n-1)(q-1)^{3} q^{e} \sqrt{q n / \alpha}} .
$$

The conclusion of the lemma follows from (4.20) and (4.25). The proof of the lemma is completed.

Proof of Proposition 4.1. It suffices to consider $x \geq 0$ since we can simply apply the result to $-W_{n, \alpha}$ when $x<0$. For a random variable $W$ with $\mathbb{E} W=0$ and $\operatorname{Var}(W)=1$, Chen and Shao (2001) proved that

$$
\sup _{x \geq 0}|\mathbb{P}(W \leq x)-\Phi(x)| \leq \sup _{x \geq 0}\left|\frac{1}{1+x^{2}}-(1-\Phi(x))\right| \leq 0.55 .
$$

Firstly, we prove (4.1). From (4.26), it suffices to prove the proposition for $n \geq 200$. Let

$$
K \geq e^{1 / 4}, q=K^{2} \max \left\{1, \frac{\alpha \log ^{2} n}{n}\right\}, \varepsilon=\frac{2 e \sqrt{2 q}}{\sqrt{n-1}} .
$$


Since $q \geq e^{1 / 2}$, it is clear that for $x>2, x / q^{x}$ is decreasing in $x$. Therefore, by applying Lemma 4.5 with noting that $q n / \alpha \geq K^{2} \log ^{2} n$, we have

$$
\begin{aligned}
\mathbb{P}_{\alpha}\left(\left|T_{n, \alpha}\right|>\frac{2 e \sqrt{2 q}}{\sqrt{n-1}}\right) \leq & \frac{q n}{\pi(q-1) q^{K e \log n} K^{2} \log ^{2} n} \\
& +\frac{n^{2} q^{3}(e(q-1) K \log n+q+1)}{\pi(n-1)(q-1)^{3} q^{e K \log n} K^{2} \log ^{4} n}:=f(q) .
\end{aligned}
$$

Since $e K \log n>5, f(q)$ is decreasing on $(1, \infty)$. Therefore

$$
\begin{aligned}
f(q) \leq f\left(K^{2}\right) & =\frac{K^{2} n}{\pi\left(K^{2}-1\right) K^{2 e K \log n} K^{2} \log ^{2} n} \\
& +\frac{n^{2} K^{6}\left(e\left(K^{2}-1\right) K \log n+K^{2}+1\right)}{\pi(n-1)\left(K^{2}-1\right)^{3} K^{2 e K \log n} K^{4} \log ^{4} n} .
\end{aligned}
$$

By choosing $K=e^{1 / 4}$ and noting that $n>200$, we have

$$
\begin{aligned}
\mathbb{P}_{\alpha}\left(\left|T_{n, \alpha}\right|>\varepsilon\right) \leq & f\left(e^{1 / 2}\right) \\
= & \frac{\sqrt{e} n}{\pi \sqrt{e}(\sqrt{e}-1) n^{0.5 e^{5 / 4} \log ^{2} n}} \\
& +\frac{n^{2} e^{3 / 2}\left(e^{5 / 4}(\sqrt{e}-1) \log n+\sqrt{e}+1\right)}{\pi(n-1)(\sqrt{e}-1)^{3} n^{0.5 e^{5 / 4}} \log ^{4} n} \\
\leq & \frac{0.05}{\sqrt{n}},
\end{aligned}
$$

and

$$
\begin{aligned}
\frac{\varepsilon}{\sqrt{2 \pi}} & =\frac{2 e^{5 / 4} \sqrt{n}}{\sqrt{\pi(n-1)}} \max \left\{\frac{1}{\sqrt{n}}, \frac{\sqrt{\alpha} \log n}{n}\right\} \\
& \leq 3.95 \max \left\{\frac{1}{\sqrt{n}}, \frac{\sqrt{\alpha} \log n}{n}\right\} .
\end{aligned}
$$

By (4.8) and (4.11), we have

$$
\begin{aligned}
\sqrt{\mathbb{E} T_{n, \alpha}^{2}} & =\sqrt{\mathbb{E}\left(\eta_{n, \alpha}^{*}-\eta_{n, \alpha}\right)^{2}} \\
& \leq \sqrt{\mathbb{E}\left(\eta_{n, \alpha}^{*}\right)^{2}}+\sqrt{\mathbb{E}\left(\eta_{n, \alpha}\right)^{2}} \\
& \leq\left(\frac{4}{3 n}+\frac{2 \alpha}{3 n(n-1)}\right)^{1 / 2}+\left(\frac{2}{n}\right)^{1 / 2} \\
& \leq\left(\left(\frac{4}{3}+\frac{2 n}{3(n-1) \log ^{2} n}\right)^{1 / 2}+\sqrt{2}\right) \max \left\{\frac{1}{\sqrt{n}}, \frac{\sqrt{\alpha} \log n}{n}\right\} .
\end{aligned}
$$

Since $n>200$, it follows from (4.32) that

$$
\left(1+\frac{\sqrt{2 \pi}}{4}\right) \sqrt{\mathbb{E} T_{n, \alpha}^{2}} \leq 4.2 \max \left\{\frac{1}{\sqrt{n}}, \frac{\sqrt{\alpha} \log n}{n}\right\} .
$$


Apply Theorem 3.2 (i), (4.1) follows from (4.30), (4.31) and (4.33).

Now we prove (4.2). If either $\delta \geq 1$ or $0<\delta<1$ and $n \leq 200$, then (4.2) holds by (4.26). Therefore we may assume that $0<\delta<1$ and $n>200$. Let

$$
0<L \leq 1, q=\frac{\alpha}{(L \delta)^{2} n}, \varepsilon^{\prime}=\frac{2 e \sqrt{2 q}}{\sqrt{n-1}} .
$$

Since $n>200$ and $0<\delta<1$, elementary calculus shows that

$$
q \geq \frac{n^{\delta}}{\delta^{2}}>51
$$

By applying Lemma 4.5, we have

$$
\mathbb{P}_{\alpha}\left(\left|T_{n, \alpha}\right|>\varepsilon^{\prime}\right) \leq \frac{\alpha}{\pi(q-1) q^{e /(L \delta)}}+\frac{(L \delta)^{2} q^{2} n \alpha(e(q-1) /(L \delta)+q+1)}{\pi(n-1)(q-1)^{3} q^{e /(L \delta)}} .
$$

By choosing $L=1$ and noting $n>200, q>51$, we have from (4.35) that

$$
\mathbb{P}_{\alpha}\left(\left|T_{n, \alpha}\right|>\varepsilon^{\prime}\right) \leq \frac{0.08 \sqrt{\alpha}}{n}
$$

and

$$
\frac{\varepsilon^{\prime}}{\sqrt{2 \pi}}=\frac{2 \sqrt{n} e}{\sqrt{\pi(n-1)} \delta} \frac{\sqrt{\alpha}}{n} \leq \frac{3.1}{\delta} \frac{\sqrt{\alpha}}{n} .
$$

Using the second inequality in (4.32) and noting again that $\alpha>n>200$, we also have

$$
\begin{aligned}
\sqrt{\mathbb{E} T_{n, \alpha}^{2}} & \leq\left(\frac{4}{3 n}+\frac{2 \alpha}{3 n(n-1)}\right)^{1 / 2}+\left(\frac{2}{n}\right)^{1 / 2} \\
& \leq\left(\sqrt{\frac{4}{3}+\frac{2 \times 201}{3 \times 200}}+\sqrt{2}\right) \frac{\sqrt{\alpha}}{n}
\end{aligned}
$$

It follows from (4.38) that

$$
\left(1+\frac{\sqrt{2 \pi}}{4}\right) \sqrt{\mathbb{E} T_{n, \alpha}^{2}} \leq 4.62 \frac{\sqrt{\alpha}}{n} .
$$

Apply Theorem 3.2 (i) with $\varepsilon^{\prime}$ plays the role of $\varepsilon$ in Theorem 3.2 (i), (4.2) follows from (4.36), (4.37) and (4.39).

The following proposition establishes non-uniform bounds on the Kolmogorov distance for Jack measures. 
Proposition 4.6. Let $n \geq 3$ be an integer. Let $p \geq 2,1 \leq \alpha<n^{2}$ and $W_{n, \alpha}$ be as in (1.5). Then for all $x \in \mathbb{R}$, we have

$$
\left|\mathbb{P}_{\alpha}\left(W_{n, \alpha} \leq x\right)-\Phi(x)\right| \leq \frac{C_{p}}{1+|x|^{p}}\left(\frac{p^{2}}{\log p}\right)^{p} \max \left\{\frac{1}{\sqrt{n}}, \frac{\sqrt{\alpha} \log n}{n}\right\}
$$

If, in addition, there exist $\delta:=\delta(\alpha, n)>0$ such that $n^{1+\delta} \leq \alpha<n^{2}$, then for all $x \in \mathbb{R}$, we have

$$
\left|\mathbb{P}_{\alpha}\left(W_{n, \alpha} \leq x\right)-\Phi(x)\right| \leq\left(1+\frac{1}{\delta^{p+1}}\right) \frac{C_{p}}{1+|x|^{p}}\left(\frac{p^{2}}{\log p}\right)^{p} \frac{\sqrt{\alpha}}{n} .
$$

Proof. We observe that if $\alpha>n^{1+\delta}, 1 / 4 \leq \delta<1$, then (4.41) implies (4.40) (the value $1 / 4$ is choosen for convenience only). Therefore, we only need to prove (4.40) for the case where $1 \leq \alpha<n^{5 / 4}$. For $n \geq 3$ and $1 \leq \alpha<n^{5 / 4}$, we have

$$
\max \left\{\frac{1}{\sqrt{n}}, \frac{\sqrt{\alpha} \log n}{n}\right\}<1 .
$$

Let $K=p+2$ and let $q, \varepsilon$ be as in (4.27). Then

$$
\varepsilon \leq 20(p+2) \max \left\{\frac{1}{\sqrt{n}}, \frac{\sqrt{\alpha} \log n}{n}\right\} .
$$

From (4.29), we have

$$
\begin{aligned}
\mathbb{P}_{\alpha}\left(\left|T_{n, \alpha}\right|>\varepsilon\right) \leq & \frac{(p+2)^{2} n}{\pi\left((p+2)^{2}-1\right) n^{2 e(p+2)}(p+2)^{2} \log ^{2} n} \\
& +\frac{n^{2}(p+2)^{6}\left(e(p+2)\left((p+2)^{2}-1\right) \log n+(p+2)^{2}+1\right)}{\pi(n-1)\left((p+2)^{2}-1\right)^{3} n^{2 e(p+2)}(p+2)^{4} \log ^{4} n} \\
\leq & \frac{C_{p}}{n^{2 e(p+2)-1}} .
\end{aligned}
$$

To apply Theorem 3.2 (ii), we also need to bound $\mathbb{E}\left|T_{n, \alpha}\right|^{2 p+2}$. Since $\left|X_{n, \alpha}\right| \leq$ $\alpha(n-1)$, we have $\left|\eta_{n, \alpha}\right| \leq \sqrt{2 \alpha}$ and therefore $\left|\eta_{n, \alpha}^{*}\right| \leq \sqrt{2 \alpha}$ (see (2.58) in Chen, Goldstein and Shao (2011)). Combining (4.42) - (4.44), we have

$$
\begin{aligned}
\mathbb{E}\left(\left|T_{n, \alpha}\right|^{2 p+2}\right) & \leq \varepsilon^{2 p+2}+(8 \alpha)^{p+1} \mathbb{P}\left(\left|T_{n, \alpha}\right|>\varepsilon\right) \\
& \leq \varepsilon^{2 p+2}+\frac{C_{p} \alpha^{p+1}}{n^{2 e(p+2)-1}} \\
& \leq C_{p} p^{2 p}\left(\max \left\{\frac{1}{\sqrt{n}}, \frac{\sqrt{\alpha} \log n}{n}\right\}\right)^{2 p+2} \\
& \leq C_{p} p^{2 p}\left(\max \left\{\frac{1}{\sqrt{n}}, \frac{\sqrt{\alpha} \log n}{n}\right\}\right)^{2} .
\end{aligned}
$$

Apply Theorem 3.2 (ii), (4.40) follows from (4.32) and (4.43)-(4.45).

To prove (4.41), we will need the following lemma. 
Lemma 4.7. If there exist $\delta>0$ such that $\alpha \geq n^{1+\delta}$, then for all $p \geq 0$, we have

$$
\mathbb{E}\left(\left|T_{n, \alpha}\right|^{p}\right) \leq \frac{C_{p} p^{p}}{\delta^{p}}\left(\frac{\sqrt{\alpha}}{n}\right)^{p} .
$$

Proof of Lemma 4.7. Let $L=1 /(p+2)$ and let $q, \varepsilon^{\prime}$ be as in (4.34). Then

$$
q \geq(p+2)^{2} \frac{n^{\delta}}{\delta^{2}}>8 \text { and } \varepsilon^{\prime} \leq \frac{10(p+2) \sqrt{\alpha}}{\delta n} .
$$

From (4.35), we have

$$
\begin{aligned}
\mathbb{P}_{\alpha}\left(\left|T_{n, \alpha}\right|>\varepsilon^{\prime}\right) & \leq \frac{q}{\pi(q-1) n^{e(p+2)-1}}+\frac{e q^{2}(q-1)+q^{2}(q+1)}{\pi(q-1)^{3}(n-1)} \frac{\alpha}{n^{e(p+2)-1}} \\
& \leq \frac{\alpha}{n^{2 p+4}} .
\end{aligned}
$$

Similar to $(4.45),(4.48)$ yields

$$
\begin{aligned}
\mathbb{E}\left(\left|T_{n, \alpha}\right|^{p}\right) & \leq\left(\varepsilon^{\prime}\right)^{p}+(2 \sqrt{2 \alpha})^{p} \mathbb{P}\left(\left|T_{n, \alpha}\right|>\varepsilon^{\prime}\right) \\
& \leq\left(\varepsilon^{\prime}\right)^{p}+\frac{C_{p} \alpha^{p / 2+1}}{n^{2 p+4}} \\
& \leq \frac{C_{p} p^{p}}{\delta^{p}}\left(\frac{\sqrt{\alpha}}{n}\right)^{p}
\end{aligned}
$$

The proof of Lemma 4.7 is completed.

Now, we will prove (4.41). Let $L, q, \varepsilon^{\prime}$ be as in the proof of Lemma 4.7. From Lemma 4.7, we have

$$
\mathbb{E}\left(\left|T_{n, \alpha}\right|^{2 p+2}\right) \leq \frac{C_{p} p^{2 p}}{\delta^{2 p+2}}\left(\frac{\sqrt{\alpha}}{n}\right)^{2 p+2} \leq \frac{C_{p} p^{2 p}}{\delta^{2 p+2}}\left(\frac{\sqrt{\alpha}}{n}\right)^{2} .
$$

Apply Theorem 3.2 (ii) with $\varepsilon^{\prime}$ plays the role of $\varepsilon$ in Theorem 3.2 (ii), (4.41) follows from (4.32), (4.48), (4.50) and the second half of (4.47).

Proofs of Theorem 1.1 and Theorem 1.3. When $\alpha \geq 1$, Theorem 1.1 is a direct consequence of Proposition 4.1. We also see that (4.1) holds if we replace $W_{n, \alpha}$ by $-W_{n, \alpha}$. To obtain Theorem 1.1 for $0<\alpha<1$, we note that from the definition of Jack measure, $\mathbb{P}_{\alpha}(\lambda)=\mathbb{P}_{1 / \alpha}\left(\lambda^{t}\right)$, where $\lambda^{t}$ is the transpose partition of $\lambda$. It also follows from (4.4) and the definition of $\alpha$-content that $W_{n, \alpha}(\lambda)=-W_{n, 1 / \alpha}\left(\lambda^{t}\right)$. Therefore

$$
\begin{aligned}
\mathbb{P}_{\alpha}\left(W_{n, \alpha}=x\right) & =\mathbb{P}_{\alpha}\left\{\lambda: W_{n, \alpha}(\lambda)=x\right\} \\
& =\mathbb{P}_{1 / \alpha}\left\{\lambda^{t}: W_{n, 1 / \alpha}\left(\lambda^{t}\right)=-x\right\} \\
& =\mathbb{P}_{1 / \alpha}\left(W_{n, 1 / \alpha}=-x\right) .
\end{aligned}
$$


From this we conclude that $\mathbb{P}_{\alpha}\left(W_{n, \alpha} \leq x\right)=\mathbb{P}_{1 / \alpha}\left(W_{n, 1 / \alpha} \geq-x\right)$. Therefore,

$$
\begin{aligned}
\sup _{x \in \mathbb{R}}\left|\mathbb{P}_{\alpha}\left(W_{n, \alpha} \leq x\right)-\Phi(x)\right| & =\sup _{x \in \mathbb{R}}\left|\mathbb{P}_{1 / \alpha}\left(W_{n, 1 / \alpha} \geq-x\right)-\Phi(x)\right| \\
& =\sup _{x \in \mathbb{R}}\left|\mathbb{P}_{1 / \alpha}\left(-W_{n, 1 / \alpha} \leq x\right)-\Phi(x)\right| \\
& \leq 8.2 \max \left\{\frac{1}{\sqrt{n}}, \frac{\log n}{\sqrt{\alpha} n}\right\} .
\end{aligned}
$$

Therefore, Theorem 1.1 also holds when $0<\alpha<1$. This completes the proof of Theorem 1.1.

When $1 \leq \alpha<n^{2}$, Theorem 1.3 is a direct consequence of Proposition 4.6. When $1 / n^{2}<\alpha<1$, the proof is similar to that of Theorem 1.1, and this completes the proof of Theorem 1.3.

\section{Appendix A}

In this Section we will prove (1.7) and two lemmas which are used in Section 2.

Proof of (1.7). For $p \geq 2$, applying Proposition 2.1 and Lemma 4.7 with noting that $\alpha \geq n^{2}$ (so that $\delta \geq 1$ ), we have

$$
\begin{aligned}
\mathbb{E}\left|W_{n, \alpha}\right|^{p} & \leq \kappa_{p}\left(1+\mathbb{E}\left|T_{n, \alpha}\right|^{p-2}\right) \\
& \leq \kappa_{p}\left(1+C_{p} p^{p}\left(\frac{\sqrt{\alpha}}{n}\right)^{p-2}\right) \\
& \leq C_{p}\left(\frac{p^{2}}{\log p}\right)^{p}\left(\frac{\sqrt{\alpha}}{n}\right)^{p-2}
\end{aligned}
$$

establishing (1.7).

Lemma A.1. Let $p>8$ and let $\kappa_{p}$ be as in Proposition 2.1, then

$$
\frac{\kappa_{p}}{\kappa_{p-2}} \geq 8\left(\frac{p-1}{\log (p-1)}\right)^{2}
$$

Proof. Let

$$
h(p)=\log \left(\kappa_{p}\right)=p(\log p-\log (\log p)+\log (7 / 4)),
$$

we have

$$
\begin{aligned}
& h(p)-h(p-2)=\int_{p-2}^{p} h^{\prime}(t) \mathrm{d} t \\
& =2+2 \log (7 / 4)+\int_{p-2}^{p} \log t \mathrm{~d} t-\int_{p-2}^{p} \log (\log t) \mathrm{d} t-\int_{p-2}^{p} \frac{\mathrm{d} t}{\log t} .
\end{aligned}
$$


Since the function $t \rightarrow \log (\log t)$ is concave,

$$
\int_{p-2}^{p} \log (\log t) \mathrm{d} t \leq 2 \log (\log (p-1)) .
$$

Next, since $p>8$, we have

$$
\int_{p-2}^{p} \frac{\mathrm{d} t}{\log t} \leq \int_{6}^{8} \frac{\mathrm{d} t}{\log t}
$$

and

$$
\begin{aligned}
\int_{p-2}^{p} \log t \mathrm{~d} t & =\int_{-1}^{1} \log (p-1+s) \mathrm{d} s \\
& =2 \log (p-1)+\int_{-1}^{1} \log \left(1+\frac{s}{p-1}\right) \mathrm{d} s \\
& =2 \log (p-1)+\int_{0}^{1} \log \left(1-\frac{s^{2}}{(p-1)^{2}}\right) \mathrm{d} s \\
& \geq 2 \log (p-1)+\int_{0}^{1} \log \left(1-\frac{s^{2}}{49}\right) \mathrm{d} s .
\end{aligned}
$$

Combining (A.3)-(A.6), numerical calculation gives

$$
\begin{gathered}
h(p)-h(p-2) \geq 2+2 \log (7 / 4)+2 \log (p-1)-2 \log (\log (p-1)) \\
\quad-\int_{6}^{8} \frac{\mathrm{d} t}{\log t}+\int_{0}^{1} \log \left(1-\frac{s^{2}}{49}\right) \mathrm{d} s \\
>2 \log (p-1)-2 \log (\log (p-1))+\log 8
\end{gathered}
$$

for all $p>8$. This implies (A.2).

Lemma A.2. Let $p>8$ and let $\kappa_{p}$ be as in Proposition 2.1. Then

$$
\frac{2(p-1)}{(1-\theta)^{p-3}} \leq \kappa_{p}
$$

where

$$
\theta=\theta(p):=\left(\frac{\log ^{2}(p-1)}{4(p-1)}\right)^{1 /(p-3)} .
$$

Proof. Firstly, we will prove that

$$
\frac{1}{1-\theta} \leq \frac{7 p}{4 \log p}
$$

which is equivalent to

$$
\frac{1}{p-3} \log \left(\frac{\log ^{2}(p-1)}{4(p-1)}\right) \leq \log \left(1-\frac{4 \log p}{7 p}\right) .
$$

imsart-generic ver. 2014/10/16 file: 2020.06.14.Jack_measures.tex date: June 16, 2020 
Since $\frac{4 \log p}{7 p}$ is decreasing when $p>8$,

$$
\frac{4 \log p}{7 p} \leq 0.1486
$$

On the other hand, it is easy to prove that

$$
\log (1-x) \geq \frac{-13 x}{12} \text { for all } 0 \leq x \leq 0.1486 .
$$

From (A.10) and (A.11), we have

$$
\log \left(1-\frac{4 \log p}{7 p}\right) \geq-\frac{13 \log p}{21 p} .
$$

Therefore, to prove (A.9), it suffices to prove that

$$
\frac{1}{p-3} \log \left(\frac{\log ^{2}(p-1)}{4(p-1)}\right) \leq-\frac{13 \log p}{21 p}
$$

which is equivalent to

$$
R_{1}(p)+R_{2}(p) \geq 0
$$

where

$$
R_{1}(p)=\frac{13 \log (p-1)}{21(p-3)}-\frac{13 \log p}{21 p}
$$

and

$$
R_{2}(p)=\frac{(8 / 21) \log (p-1)-2 \log (\log (p-1))+\log (4)}{p-3} .
$$

Elementary calculus shows that $R_{1}(p) \geq 0$ and $R_{2}(p) \geq 0$ for all $p>8$. Therefore (A.13) holds, completing the proof of (A.8).

Now, we will prove (A.7). Since $p>8$, we have from (A.8) that

$$
\frac{2(p-1)}{(1-\theta)^{p-3}} \leq 2(p-1)\left(\frac{4 \log p}{7 p}\right)^{3}\left(\frac{7 p}{4 \log p}\right)^{p} \leq \frac{(\log 8)^{3}}{196}\left(\frac{7 p}{4 \log p}\right)^{p}=\kappa_{p} .
$$

The proof of the lemma is completed.

\section{Acknowledgements}

The first author and the third author were partially supported by Grant R146-000-182-112 and Grant R-146-000-230-114 from the National University of Singapore. A substantial part of this paper was written when the third author was at the Institute for Mathematical Sciences (IMS) and Department of Mathematics, National University of Singapore. He would like to thank the IMS staff and the Department of Mathematics for their hospitality. 


\section{References}

Baik, J., Deift, P., and Johansson, K. (1999) On the distribution of the length of the longest increasing subsequence of random permutations. J. Amer. Math. Soc. 12, no. 4, 1119-1178. MR1682248

Borodin, A., Okounkov, A., and Olshanski, G. (2000) Asymptotics of Plancherel measures for symmetric groups. J. Amer. Math. Soc. 13, 481-515. MR1758751

Chatterjee, S., and Dey. P. S. (2010) Applications of Stein's method for concentration inequalities. Ann.Probab. 38, no. 6, 2443-2485. MR2683635

Chen, L. H. Y., and Shao, Qi-Man. (2001) A non-uniform Berry-Esseen bound via Stein's method. Probab. Theory Related Fields. 120, no. 2, 236-254. MR1841329

Chen, L. H. Y., and Shao, Q. M. (2004) Normal approximation under local dependence. Ann. Probab. 32, no. 3A, 1985-2028. MR2073183

Chen, L. H. Y., Goldstein, L., and Shao, Q. M. (2011) Normal approximation by Stein's method. Probability and its Applications (New York). Springer, Heidelberg. MR2732624

Chen, L. H. Y., Goldstein, L., and Röllin, A. (2020) Stein's method via induction. https://arxiv.org/abs/1903.09319

Diaconis, P., and Shahshahani, M. (1981) Generating a random permutation with random transpositions. Z. Wahr. Verw. Gebiete 57, no.2 , 159-179. MR0626813

Dołęga, M., and Féray, V. (2016) Gaussian fluctuations of Young diagrams and structure constants of Jack characters. Duke Math. J. 165, no. 7, 1193-1282. MR3498866

Dołęga, M., and Śniady, P. (2019) Gaussian fluctuations of Jack-deformed random Young diagrams. Probab. Theory Related Fields 174, no. 1-2, 133-176. MR3947322

Eskin, A., and Okounkov, A. (2001) Asymptotics of numbers of branched coverings of a torus and volumes of moduli spaces of holomorphic differentials. Invent. Math. 145, no. 1, 59-103. MR1839286

Frobenius, G. F. (1900) Uber die Charaktere der symmetrischen Gruppe. Sitz. Konig. Preuss. Akad. Wissen, 516-534; Gesammelte abhandlungen III, Springer-Verlag, Heidelberg, 1968, 148-166.

Fulman, J. (2004) Stein's method, Jack measure, and the Metropolis algorithm. J. Combin. Theory Ser. A 108, no. 2, 275-296. MR2098845

Fulman, J. (2006) An inductive proof of the Berry-Esseen theorem for character ratios. Ann. Comb. 10, no. 3, 319-332. MR2284273

Fulman, J., and Goldstein, L. (2011) Zero biasing and Jack measures. Comb. Probab. Comput. 20, no. 5, 753-762. MR2825589

Goldstein, L. (2005) Berry-Esseen bounds for combinatorial central limit theorems and pattern occurrences, using zero and size biasing. J. Appl. Probab. 42, no. 3, 661-683. MR2157512

Goldstein, L., and Reinert, G. (1997) Stein's method and the zero bias transformation with application to simple random sampling. Ann. Appl. Probab. 7, no. 4, 935-952. MR1484792 
Hora, A. (1998) Central limit theorem for the adjacency operators on the infinite symmetric group. Comm. Math. Phys. 195, no. 2, 405-416. MR1637801

Hora, A., and Obata, N. (2007) Quantum probability and spectral analysis of graphs. Theoretical and Mathematical Physics. Springer, Berlin. MR2316893

Ibragimov, R. and Sharakhmetov, Sh. (1997) On an exact constant for the Rosenthal inequality. Theory Probab. Appl. 42, no. 2, 294-302. MR1474714

Johansson, K. (2001) Discrete orthogonal polynomial ensembles and the Plancherel measure. Ann. of Math. (2) 153, no. 1, 259-296. MR1826414

Johnson, W. B., Schechtman, G., and Zinn, J. (1985) Best constants in moment inequalities for linear combinations of independent and exchangeable random variables. Ann. Probab. 13, no. 1, 234-253. MR0770640

Kerov, S. (1993) Gaussian limit for the Plancherel measure of the symmetric group. C. R. Acad. Sci. Paris Ser. I Math. 316, no. 4, 303-308. MR1204294

Kerov, S. (2000) Anisotropic Young diagrams and Jack symmetric functions. Funct. Anal. Appl. 34, no. 1, 41-51. MR1756734

Latała, R. (1997) Estimation of moments of sums of independent real random variables. Ann. Probab. 25, no. 3, 1502-1513. MR1457628

Matsumoto, S. (2008) Jack deformations of Plancherel measures and traceless Gaussian random matrices. Electron. J. Combin. 15, no. 1, research paper 149, 18 pp. MR2465773

Rosenthal, H. P.(1970) On the subspaces of $L^{p}(p>2)$ spanned by sequences of independent random variables. Israel J. Math. 8, 273-303. MR0271721

Sagan, B. (2001) The symmetric group. Representations, combinatorial algorithms, and symmetric functions. 2nd edn. Springer-Verlag, New York. MR1824028

Shao, Q. M., and Su, Z. G. (2006) The Berry-Esseen bound for character ratios. Proc. Amer. Math. Soc. 134, no. 7, 2153-2159. MR2215787

Śniady, P. (2006) Gaussian fluctuations of characters of symmetric groups and of Young diagrams. Probab. Theory Related Fields 136, no. 2, 263-297. MR2240789

Stein, Charles. (1986) Approximate computation of expectations. Institute of Mathematical Statistics Lecture Notes Monograph Series, 7. Institute of Mathematical Statistics, Hayward, CA. iv+164 pp. MR0882007 